
June 1939

\title{
NATURE OF ENERGY LOSSES IN AIR CAPACITORS AT LOW FREQUENCIES
}

\author{
By Allen V. Astin
}

ABSTRACT

The power factors of a number of types of air capacitors have been observed in the frequency range from 60 to $1,000 \mathrm{c} / \mathrm{s}$. The method of measurement permitted evaluation of true, or "absolute," power-factor values as small as $5 \times 10^{-7}$. In guard-ring air capacitors the losses occur on the surfaces of the electrodes and give rise to a power factor which is proportional to capacitance (inverse electrode separation). The same type of losses in addition to the losses in the solid insulation, are evident in two-electrode capacitors.

For most two-electrode air capacitors of variable capacitance, $C$, the loss may be represented by the function $A+B / C$, where $A$ depends upon the electrode material, humidity, and frequency, and $B$ depends upon the insulation material, humidity, and frequency.

Of the 10 metals used as electrode materials in guard-ring capacitors, aluminum gave the greatest power factor and silver plate the smallest. The power factors for the other materials, which included brass, monel metal, stainless steel, nickel, and brass plated with gold, rhodium, and chromium, were only slightly different from that for silver-plated brass, but they were appreciably smaller than the values for aluminum. The power factor of a guard-ring capacitor is a function of humidity and shows a maximum value at relative humidities between 40 and 70 percent at low frequencies. The maximum value is apparently due to a variable conductivity of the moisture layer with thickness.

The loss which occurs in the electrode surface layer is also a function of the homogeneity of the electric field. The nonuniform field in a three-electrode rotating-plate capacitor gives rise to a power factor which decreases as the capacitance is increased.

\section{CONTENTS}

Page

I. Introduction _...

II. Calibration of standard for power factor

III. Power factors of two-electrode capacitors

IV. Power factors of three-electrode capacitors _._.

V. Contribution of a surface layer to power factor

VI. Effect of a nonhomogeneous field on power factor

VII. Conclusion_._.

VIII. References

\section{INTRODUCTION}

Previous investigations into the causes of energy losses in air capacitors have in general disclosed four major contributing factors. As summarized by Field and Sinclair [1] ${ }^{1}$ these are: (1) a conductance and dielectric absorption loss in the solid insulation; (2) a dielectric absorption loss in dust or lint which sometimes accumulates on the plates of a capacitor; (3) an eddy-current loss in the metallic electrodes; and (4) a loss due to the effective resistance of the leads and electrodes and the contact resistance to the rotating shaft of a variable capacitor. The energy losses from the two latter factors

1 Figures in brackets indicate the literature references at the end of this paper. 
decrease with decreasing frequency and for frequencies below 1,000 $\mathrm{c} / \mathrm{s}$ are quite negligible. The two remaining factors are, however, inadequate to explain recent observations with high-precision bridges at low frequencies $[2,3,4]$. In the observations reported in these references the effects of losses in both solid insulation and dust were eliminated, but losses were still found in the capacitors. These losses, which have been shown to depend upon the electrode surface, electrode separation, and the frequency, may give rise to power factors of $1 \times 10^{-5}$ or larger. In order to obtain more information concerning the nature of these losses, a number of guard-ring capacitors have been constructed of various electrode materials and their power factors measured under a wide range of conditions. The power factors were measured by the bridge method described in a previous publication [2], which permitted the determination of the true, or absolute, values in contrast to the relative or difference values which are ordinarily obtained by bridge methods. The precision of the method allowed the evaluation of power factors as small as $5 \times 10^{-7}$ in the commercial and audio-frequency range.

This method of power-factor evaluation has also been used for observations on the power factors of variable, two-electrode air capacitors. Previously, such measurements have been limited to power-factor-difference methods. From these measurements it has been possible to obtain evidence of the existence of the same type of loss factor that is predominant in a guard-ring capacitor, namely, a loss originating on the surface of the metallic electrodes.

\section{CALIBRATION OF STANDARD FOR POWER FACTOR}

A three-electrode rotating-plate capacitor has been used as a working standard of power factor and capacitance for all measurements which are included in this paper. This capacitor, which has been described in detail previously [2], has nickel-plated brass electrodes, $3 \mathrm{~mm}$ apart when interleaved, and a capacitance range of $1,800 \mu \mathrm{f}$. The third electrode permitted the elimination from the measured power factor of the capacitor of any losses in its insulating supports. Although this same result could have been obtained more readily with a guard-ring capacitor, the rotating-plate capacitor proved to be a much more satisfactory working standard for bridge measurements because of the greater ease with which its capacitance could be varied. As indicated above, the power factor of a threeelectrode capacitor cannot be assumed equal to zero; hence some other means must be available in order to obtain actual power-factor values. This can be accomplished with the aid of a guard-ring capacitor of adjustable electrode spacing (variable capacitance). As pointed out in the reference previously cited [2], the power factor of such a capacitor is proportional to its capacitance; hence at zero capacitance its power factor would be zero. By determining the rate of change of power factor of a guard-ring capacitor with capacitance and extrapolating to zero capacitance, actual power-factor values may be obtained.

In applying this principle to the power-factor calibration of the three-electrode rotating-plate capacitor it was simpler, because of the greater ease of capacitance adjustment of the rotating-plate capacitor, first to determine its rate of power-factor change with capacitance. Then by using measured differences in the power factors at various 
capacitance settings of the rotatingplate capacitor and an adjustable guard-ring capacitor, the rate of power factor change of the latter instrument was determined and extrapolated to zero capacitance. The details of this procedure may be seen by referring to figure $1(a, b$, c), where the curves $C_{g}-C_{s}$ represent the power-factor differences at the indicated frequencies of an adjustable guard-ring capacitor, $C_{g}$, and the three-electrode rotatingplate capacitor, $C_{s}$, at equal capacitance settings. The $C_{s}$ curves represent the power-factor variations of $C_{s}$ with capacitance. The ordinates of these curves are fixed arbitrarily by the doubly circled points which represent the powerfactor differences, at the indicated frequencies, of $C_{s}$ and a fixed cylindrical guard-ring capacitor, $C_{0}$, having silver-plated electrodes $1 \mathrm{~cm}$ apart. The power factor of $C_{0}$ thus provides a tentative reference point. The change in power factor with capacitance of $C_{s}$ with respect to this point was determined by making power-factor-differencemeasurements with auxiliary capacitors individually and in parallel. ${ }^{2}$

The detail of this procedure is as follows: Let $\phi_{s 1}$ be the phase-defect angle, or power factor, of $C$, at a particular scale setting. Let $\phi_{A 1}$ and $\phi_{B 1}$ be the power factors of two other capacitors having the same capacitance as $C_{s}$ at its setting corresponding to $\phi_{s 1}$. Then the power-factor differences, $\phi_{n_{1}}-\phi_{B 1}=M_{1}$ and $\phi_{s 1}-\phi_{B 1}=M_{2}$, may be measured in the bridge. The two auxiliary capacitors are then connected in parallel, and it is a simple matter to show that the power factor of the combination will be the arithmetical mean of their individual values. The capacitance of $C_{8}$ is then varied to equal that of the combined auxiliaries and power-factor difference, $\phi_{B 2}-\left(\phi_{A 1}+\phi_{B 1}\right) / 2=M_{3}$, measured. Here, $\phi_{s 2}$ is the power factor of $C_{s}$ at its new scale setting. Combining the values for $M_{1}, M_{2}$, and $M_{3}$, it is found that

$$
\phi_{s 1}-\phi_{s 2}=\left(M_{1}+M_{2}\right) / 2-M_{3} \text {, }
$$

which represents the change in power factor of $C_{\mathrm{s}}$ between two scale settings. Additional points may be obtained by the use of a larger number of auxiliary capacitors.

FIGURE 1.-Power-factor differences and true power-factor values of a variable guard-ring capacitor, $C_{0}$, and a threeelectrode rotating-plate capacitor, $C_{\mathbf{s}}$, as functions of capacitance.

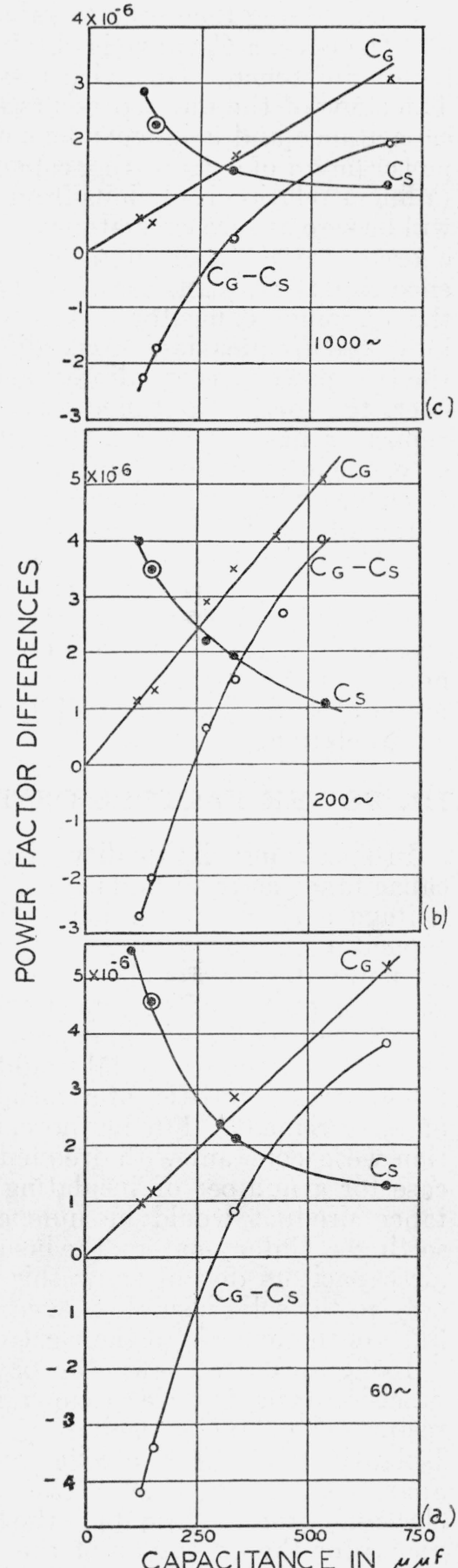

(c)

CAPACITANCE IN $\mu \mu f$ 
Upon adding the ordinate values of a $C_{s}$ curve to the corresponding $C_{g}-C_{s}$ curve, a $C_{g}$ curve is obtained which thus represents the powerfactor differences of $C_{g}$ with respect to the fixed power factor of $C_{0}$. The slope of the line represents the rate of power-factor change with capacitance and is independent of the power factor of $C_{0}$. Since the power factor of a guard-ring capacitor is proportional to its capacitance (a linear relation is obvious from fig. $1(\mathrm{a}, \mathrm{b}, \mathrm{c})$ ), the power factor of $C_{g}$ will be zero at zero capacitance. The line will intercept the arbitrarily chosen zero of the ordinate scale only if the power factor of the reference capacitor, $C_{0}$, is zero. It happens in this case that the value of the reference capacitor is zero or at least less than $5 \times 10^{-7}$ at the indicated frequencies. Accordingly, the curves $C_{0}$ and $C_{s}$ represent the true power-factor calibration curves for the two capacitors. However, the method outlined above is applicable even if the reference capacitor has a power factor greater than zero. If such were the case, the intercept of $C_{g}$ curve would be below the origin, and both the $C_{0}$ curve and $C_{s}$ curve would have to be shifted upward by the amount of the intercept to give true power-factor values. It should be pointed out that the proportional relation between the power factor and capacitance of a guard-ring capacitor is assumed from the linear relation, because it has been found that the zero value obtained for $C_{0}$ by this process is independent of (1) the frequency, (2) the air pressure (below normal atmospheric pressure) in either $C_{0}$ or $C_{g}$, and (3) the electrode material and dimensions of the guard-ring capacitor used for the extrapolation.

\section{POWER FACTORS OF TWO-ELECTRODE CAPACITORS}

In specifying the quality of a two-electrode capacitor of variable capacitance as regards its freedom from power loss under alternating voltage a single expression would be desirable. If the losses in a capacitor were confined entirely to solid insulators in a constant field, its power factor would be inversely proportional to its capacitance giving a power-factor-capacitance product which would be constant for all scale settings. This product, which has been called the "figure of merit" of a capacitor [5] would then be a satisfactory designation of the loss characteristics of a variable air capacitor under the conditions of measurement. Furthermore, if the loss per cycle in the solid insulation were constant with frequency as Chaffe [6] has shown to be the case for a number of insulating materials, the power-factor-capacitance product would be independent of both frequency and scale setting. Unfortunately, the insulators in the best commercial variable air capacitors do not show this property nor are the losses confined only to the solid insulation even at low frequencies when the dust and lint are removed from the electrode surfaces.

In figure 2 are shown the power factors at three frequencies of a precision-type, two-electrode variable air capacitor plotted against the reciprocal of the capacitance. The insulation of the capacitor is Isolantite and the electrodes are of aluminum spaced about $1 \mathrm{~mm}$ apart when interleaved. The capacitor is of the common rotatingelectrode type. The data in the figure show that neither of the requirements for the constancy of the power-factor-capacitance product is met with this particular capacitor. The power-factor change with 
frequency is appreciable and the power factor at each frequency, although linear with the reciprocal of capacitance, is definitely not proportional to it. The finite values of the intercepts on the ordinate axis indicate that a constant must be added to the power factor in order to adequately represent the power-factor variations at a particular frequency.

The results of power-loss observations on a large number of variable air capacitors have indicated that in no case is an equivalent parallel resistance sufficient to represent the losses at one frequency. The

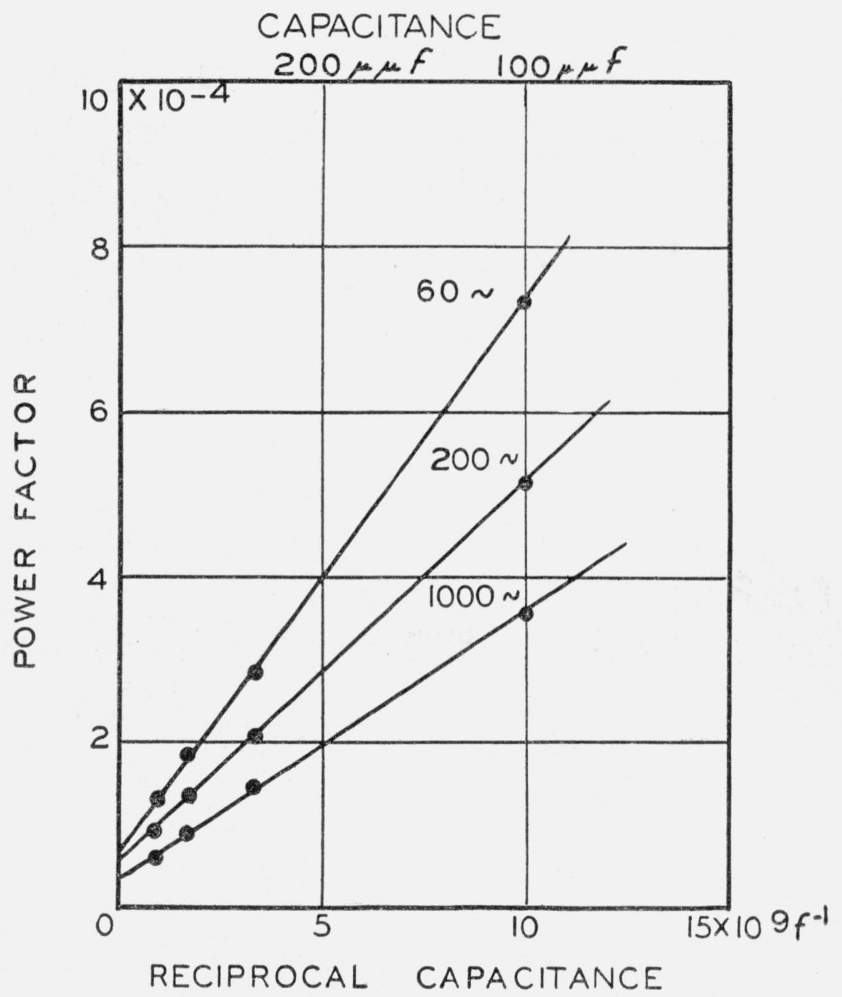

FiguRE 2.-Power factors at three frequencies of a precision type, two-electrode variable air capacitor with Isolantite insulation.

Relative humidity about 50 percent.

power factors at 60 cycles of five representative capacitors are shown in figure 3. Curve A represents a National Bureau of Standards type capacitor [7] with fused-silica and amber insulation and brass electrodes about $2.5 \mathrm{~mm}$ apart. The power-factor values for $60 \mathrm{c} / \mathrm{s}$ shown in figure 2 are shown again by $B$, and $D$ represents the values for another capacitor of identical type. The curve $C$ represents a capacitor having Isolantite insulation and brass electrodes about 1 $\mathrm{mm}$ apart, and $E$ is for a capacitor similar to that of $C$, except that the insulation is of clear fused quartz. In each case the power factor is (1) a linear function of the reciprocal of the capacitance and, (2) finite and positive at the extrapolated value for infinite capacitance. 
The power factors for each of the capacitors shown in figure 3 can be represented by the function

$$
A+B / C,
$$

where $A$ is the intercept on the power-factor axis and $B$ is the slope of the curve. The part of the power factor represented by the $B / C$ term may be attributed to the insulation, assuming $B=1 / \omega R$, where

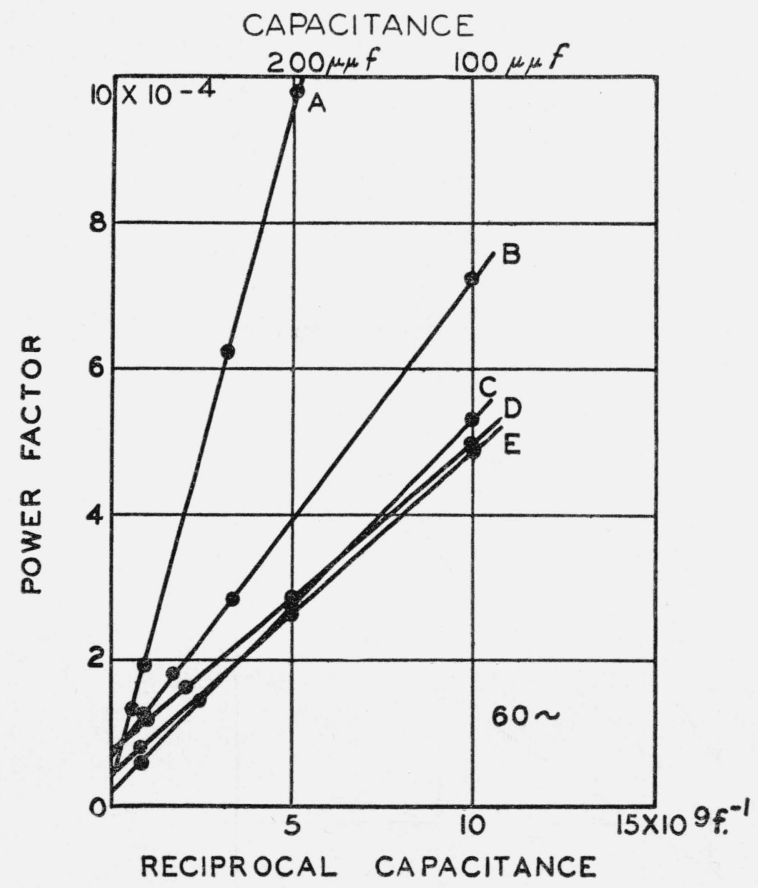

FIgURE 3.-Power factors of five variable air capacitors with solid insulation observed at $60 \mathrm{c} / \mathrm{s}$ and 50 percent relative humidity.

Insulation and electrode material of the capacitors were as follows: $A$, fused-silica and amber insulation and brass electrodes; $B$ and $D$, Isolantite insulation and aluminum electrodes; $C$, Isolantite insulation and brass electrodes; $E$, clear fused-quartz insulation and brass electrodes.
$R$ is the equivalent parallel resistance of the insulation (in general $R$ is a function of frequency) and $\omega$ is $2 \pi$ times the frequency. The part represented by the $A$ term may be explained by assuming a uniform surface layer on the electrodes, which gives rise to an equivalent resistance, $r$, in series with the capacitance. The power factor produced by such a resistance would be $\omega r C$, but since $r$ would be inversely proportional to $C$ (electrode area), this powerfactor term would be independent of scale setting. It should be noted that values of $A$ are less for the capacitors with brass electrodes than for those with aluminum electrodes.

An explanation of the decrease of $A$ with frequency (fig. 2) requires a consideration of the capacitance of the surface layer as well as its resistance. A more complete discussion of this effect, which produces the predominant power factor of a guard-ring capacitor, will be given in a later section of this paper.

The variation of the equivalent parallel resistance, $R$, with frequency is shown in figure 4 for three of the capacitors represented in figure 3 . The values for the $B$ curve were computed from the slopes of the three straight lines of figure 2 , using the relation $B=1 / \omega R$, where $B$ represents the slope for a particular frequency. The curves $A$ and $E$ were similarly obtained. The values indicated for zero frequency represent actual d-c resistance values obtained from the voltage-current ratio 1 minute after applying the voltage to the capacitor. The dashed curves represent the theoretical resistance values, which are inversely proportional to frequency, that is, the type of variations 
to be expected if the power factor represented by the equivalent parallel resistance were independent of frequency. The curves were made to pass through the experimental points for $1,000 \mathrm{c} / \mathrm{s}$. The curve $A$ follows this type of variation quite closely, but $B$ and $E$, representing capacitors having appreciably lower power factors, depart appreciably from it. The amount of departure is especially indicated by the zero frequency values (d-c resistances) at which point the theoretical curve approaches infinity. Because of the differences in the frequency variation of these resistances, the anomaly is presented of the capacitor having the largest power factor also having the highest d-c resistance and vice versa. This of course is merely coincidental for the particular three capacitors under observation and should not be taken as a generality but rather as a proof that the d-c performance of a capacitor offers little if any information as to its a-c power factor.

All of the data represented in figures 2,3 , and 4 were taken at approximately the same temperature and relative humidity, namely, $27^{\circ} \mathrm{C}$ and 50 -percent relative humidity. This was essential for proper comparison of the power factor of the different capacitors. The temperatures normally

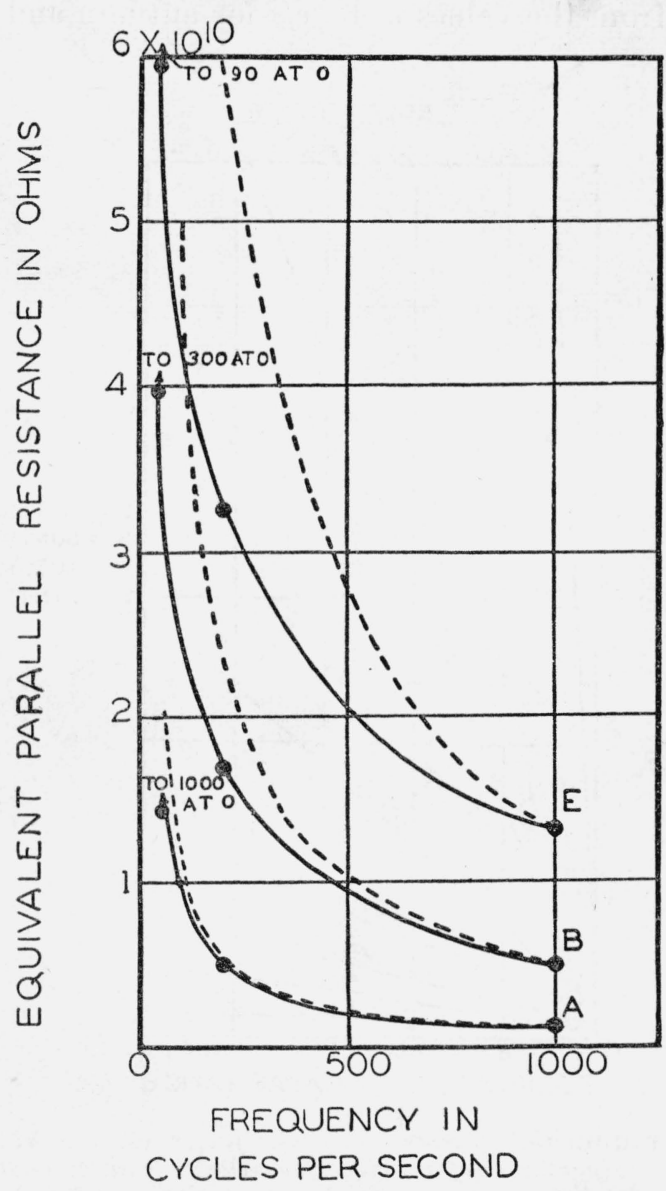

FIgURe 4.-Equivalent parallel resistances of three air capacitors as a function of frequency.

Zero values represent 1-minute d-c resistances. Dashed lines represent theoretical resistance values below $1,000 \mathrm{c} / \mathrm{s}$ which are inversely proportional to frequency. Curve designations $A, B$, and $E$ correspond to figure 2 . observed throughout a year in the capacitance laboratory of the National Bureau of Standards vary between $24^{\circ}$ and $31^{\circ} \mathrm{C}$, which range is sufficiently restricted to produce only slight differences in the power factors of capacitors. However, the seasonal relative humidity, which may range from 15 to 85 percent, causes enormous fluctuations in the power factors of the capacitors. An indication of the spread of values which may be obtained at different humidities is given in figure 5, which shows available data on a capacitor with quartz insulation and brass electrodes (the same capacitor as represented by curves $E$, fig. 3 and 4 ). 
The summer values were taken when the room humidity was at 85 percent, the autumn and spring values at 50 percent, and the winter values at 35 percent. Because of the partial sealing of the insulation in the case of the capacitor, considerable lag was observed in the variations of power factor with humidity changes. This is evident from the values at $60 \mathrm{c} / \mathrm{s}$ for autumn and spring, both of which were

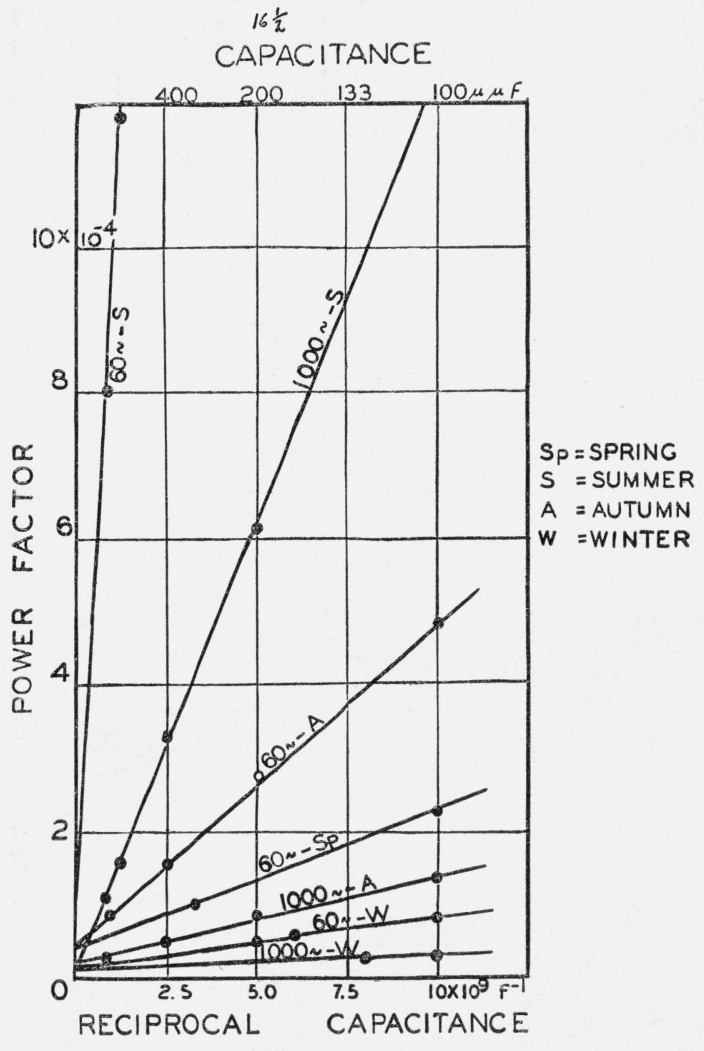

FigURe 5.-Curves of power factor vs. reciprocal capacitance of a variable, two-electrode air capacitor at different seasons (different relative humidities). taken at about 50-percent relative humidity.

It is obvious from the data of figure 5 that any specification concerning the a-c losses in a capacitor of this type would be quite useless unless the insulation of the capacitor were in equilibrium with an atmosphere of clearly defined relative humidity. Likewise, it would be impossible to use such a capacitor as a reference standard for power-factor difference measurements unless proper.humidity stability were preserved. It should, however, be pointed out that the range of variations with Isolantite insulation is generally less than that shown in figure 5 , the winter values being higher and the summer values lower. The range of values would probably be appreciably lowered by a careful cleaning of the quartz, since the data shown were taken with the capacitor in the same condition as when received from the manufacturer. From the data so far presented it would appear quite safe to draw the conclusion that the power factor of a two-electrode, variable air capacitor may be represented by the expression $A+B / C$, where $A$ is a function of frequency, humidity, and the properties of the electrode surface material and $B$ is a function of frequency, humidity, and the properties of the insulation material. In general, $A$ and $B$ would appear to decrease with increasing frequency and increase with increasing humidity. Although the expression $A+B / C$ has been found adequate to represent the power factors in most cases, exceptions have been encountered, particularly when the $A$ term is the predominant loss term. Under such circumstances $A$ may not be a constant (at a fixed frequency and humidity) because of variations in 
the surface of the electrodes or because stray capacitance increases the effective electrode separation at low capacitance values. Two extreme cases are shown in figure 6. The curve $(a)$ is for a capacitor with very good quartz insulation in a low-humidity atmosphere, and somewhat tarnished brass electrodes of 1-mm spacing. The change of slope is very likely due to variations in the electrode surface as the electrodes are rotated for different capacitance settings. The curve (b) represents the values for a capacitor with Isolantite insulation and

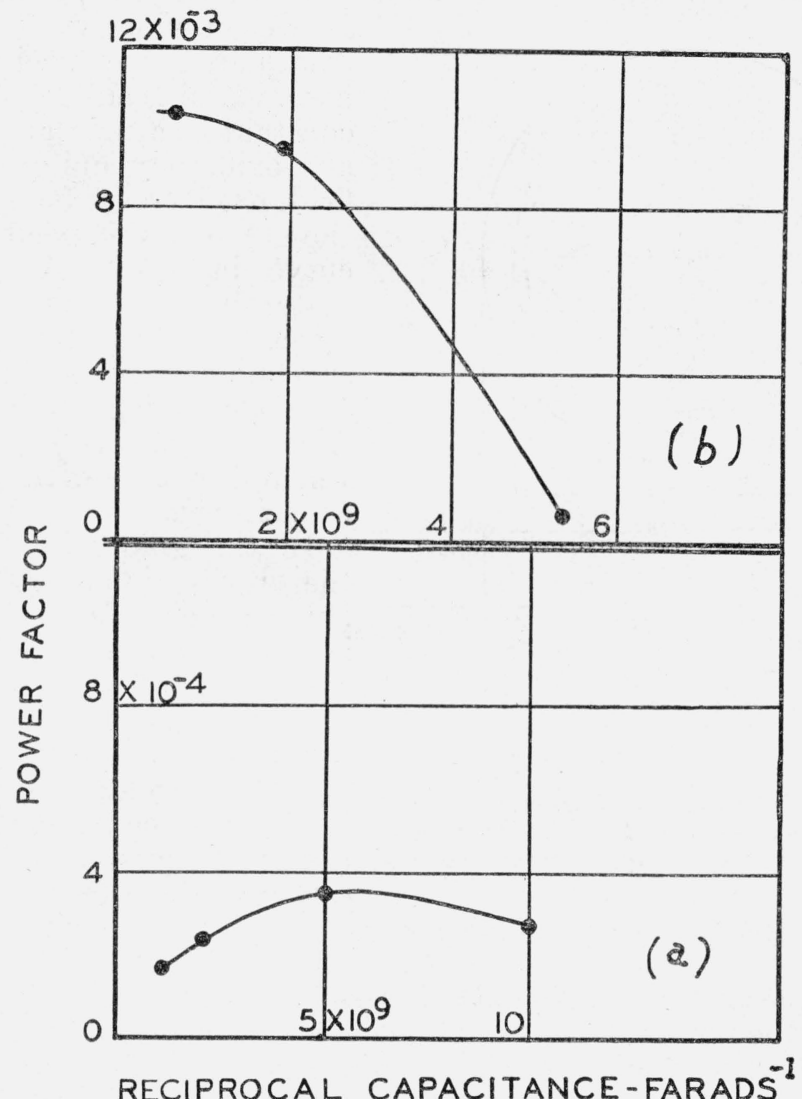

FIGURE 6.--Power factors of two variable capacitors in which the electrode surface loss is predominant.

Observations were made at a frequency of $60 \mathrm{c} / \mathrm{s}$.

cadmium-plated electrodes. The plating was extremely heavy and flaky and comparable in thickness to the electrode separation $(0.5$ $\mathrm{mm}$ ) and gave rise to the equivalent of a large resistance in series with the capacitance. As the capacitance was decreased the relative effect of stray capacitance, which increases the effective electrode separation of the capacitor, increased, causing a decrease in power factor. Such power-factor behaviors are exceptional, but the possibility of the existence of such is sufficient to require the qualification that the $A+B / C$ type of variation is not followed unless the surface losses are small and uniform. 


\section{POWER FACTORS OF THREE-ELECTRODE CAPACITORS}

When the power factor of a three-electrode capacitor is measured in an a-c bridge with a Wagner ground, the effect of losses in the solid insulators which support and separate the electrodes may be eliminated. The three-electrode capacitor may be of the guard-ring type or it may have rotor and stator assemblies with a third or guard electrode for carrying the insulating supports. The power-factorcapacitance characteristics of both types of capacitors have been

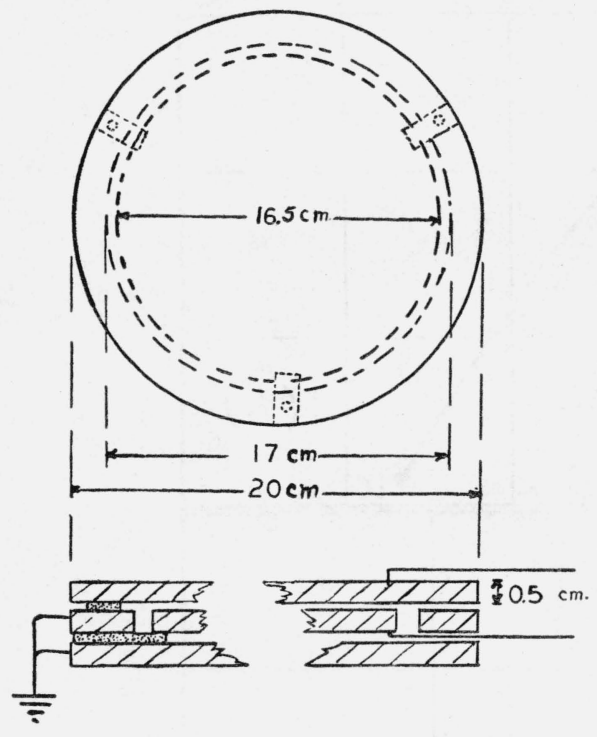

FIGURE 7.-Plan and section of the guardring capacitors used to investigate power factor as a function of electrode material and relative humidity.

Electrical connections are indicated in the sectional view. shown previously in figure 1 . The guard-ring capacitor, which has a variable electrode spacing, constant electrode area, and an approximately uniform electric field, has a power factor proportional to its capacitance $\left(C_{\theta}\right.$ curves in fig. 1). The rotating plate, guard-electrode capacitor, which has a constant electrode spacing, variable electrode area, and partially nonuniform electric field, has a power factor which decreases with increasing capacitance.

In the guard-ring capacitor the proportional relationship between power factor and capacitance can be most simply represented by an equivalent resistance, $r$, in series with the capacitance, $C$. The power factor of such a combination is $\omega r c$. Previous evidence [2] has indicated that the equivalent resistance, $r$, is due to a surface layer on the electrodes. Accordingly, for a given electrode material and conditions of measurement, $r$ would beinversely proportional to the electrode area, and the power factor would be independent of the electrode area. The manner in which $r$ is related to the properties of a hypotethical surface layer and the frequency will be treated in detail in section V. An explanation of the power-factor characteristics of the rotating-plate capacitor involves a consideration of the nonhomogeneous electric field at the edges of the electrodes and will be given in section VI.

The effect of electrode material and of the measuring conditions on the power factor of a guard-ring capacitor has been studied by preparing capacitors of 10 different materials and by measuring their power factors at various frequencies, temperatures, and relative humidities. The construction of the capacitors is shown in figure 7 . These were placed in a sealed air bath in which both the temperature and relative humidity could be controlled. It was found that the losses depended considerably upon the relative humidity, but no definite dependence upon temperature alone in the available range of $20^{\circ}$ to $40^{\circ} \mathrm{C}$ could be determined. Accordingly, all the results at various humidities are given for a single temperature, namely, $30^{\circ} \mathrm{C}$. 
Electrodes were made of the following materials: brass, aluminum, monel metal, nickel, and stainless steel. In addition, capacitors of brass were plated with the following metals: gold, silver, nickel,

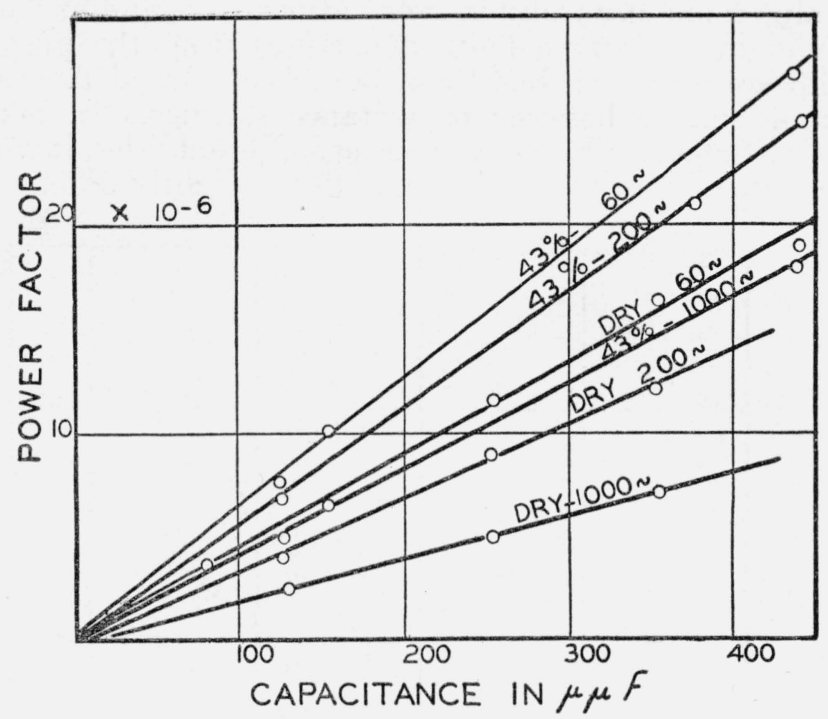

FIgURE 8.-Power factors of a guard-ring air capacitor with aluminum electrode of variable spacing (variable capacitance).

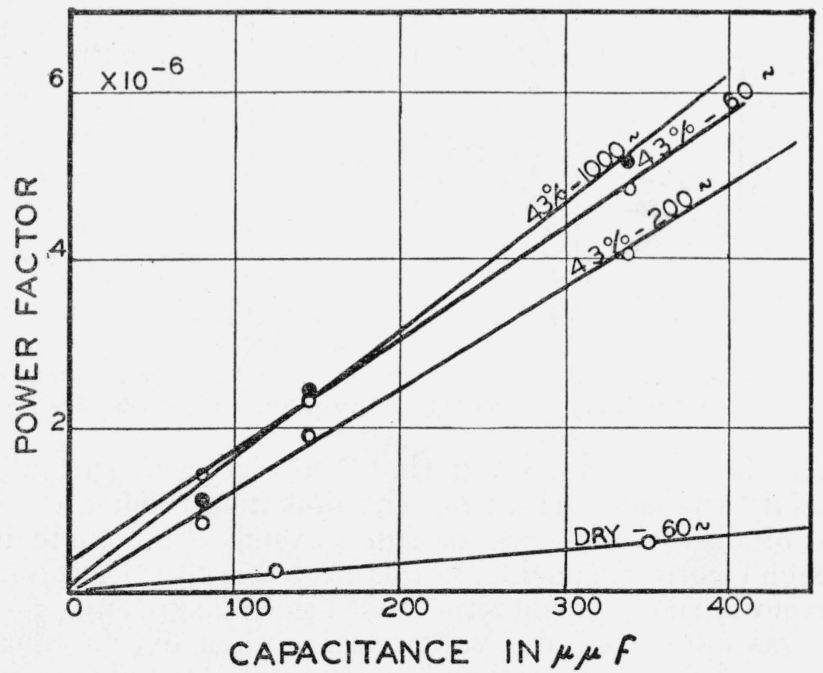

FIGURE 9.-Power factors of a guard-ring air capacitor with brass electrodes of variable spacing (variable capacitance).

chromium, and rhodium, and a capacitor of aluminum was anodized. The solid electrodes were ground smooth with fine emery paper and crocus cloth, and all electrodes were cleaned with soap and water and then rinsed thoroughly with water.

The results on aluminum and brass electrodes, the two metals most commonly used for electrodes in capacitors, are shown in figures 8 
and 9, for a dry atmosphere and an atmosphere of 43-percent relative humidity. The proportional relationship between power factor and capacitance is again apparent. In figure 10 are shown the results for anodized aluminum electrodes in a dry atmosphere and at 85-percent relative humidity. Here definite departures from the proportional relationship are observed, but these have been traced to changes in the electrode surface between capacitance settings. In taking observations on these electrodes over a range of humidities, it was found that upon returning to the initial humidity a slightly different power

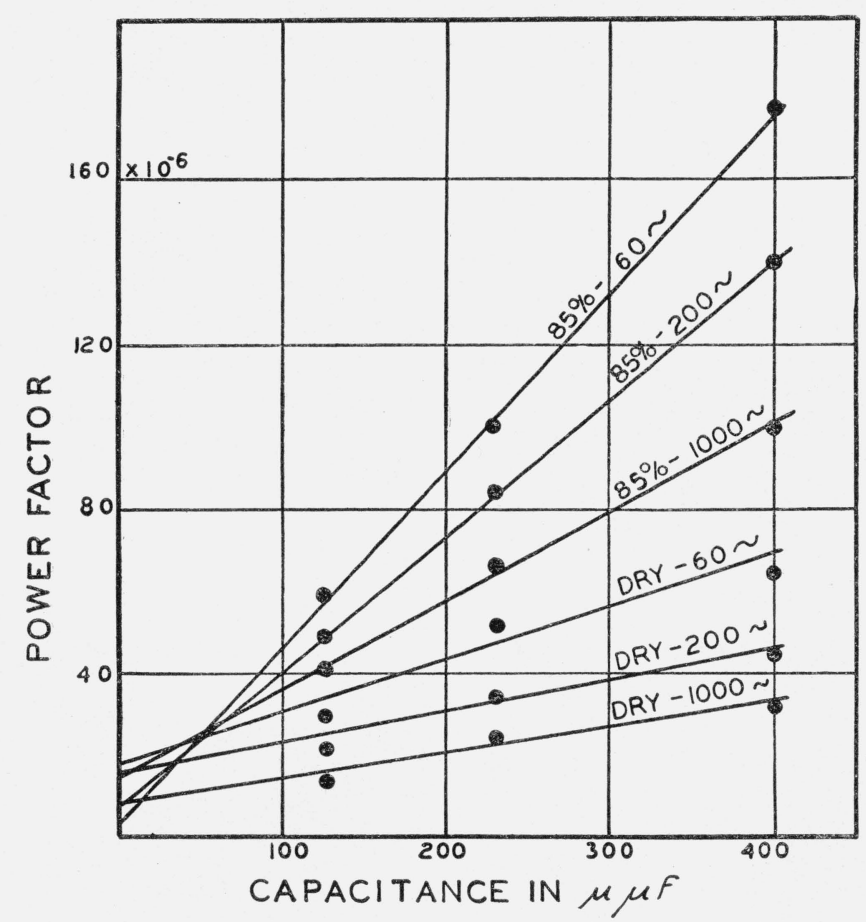

FIGURe 10.-Power factors of a guard-ring air capacitor with anodized aluminum electrodes of variable spacing (variable capacitance).

factor was obtained, indicating that the electrode surface was no longer exactly the same as during the first measurement. Since the procedure of observation was to run through a complete humidity cycle at each electrode spacing, it was probable that the same surface conditions no longer existed at the next electrode spacing. When the humidity was kept fixed and values were taken over a range of capacitances, a strictly proportional relationship was always observed. The measured power factors seem to indicate that the effect of the humidity cycle on the surface condition is more of the nature of a permanent change than a hysteresis type effect. Definite indication of a slow change in the nature of an aluminum surface with time is given in figure 11, which shows groups of observations at $60 \mathrm{c} / \mathrm{s}$ taken several months apart. The earliest set of data was taken shortly after the grinding of the surfaces, and nothing was done to the surfaces between then and the subsequent measurements except washing 
and rinsing. The gradual increase in the slopes of the curves with time probably represents a growth of the oxide layer on the electrodes. The scattering of points from the lines is greater than for a rapid continuous series of observations and is to be expected when the effect being measured is varying.

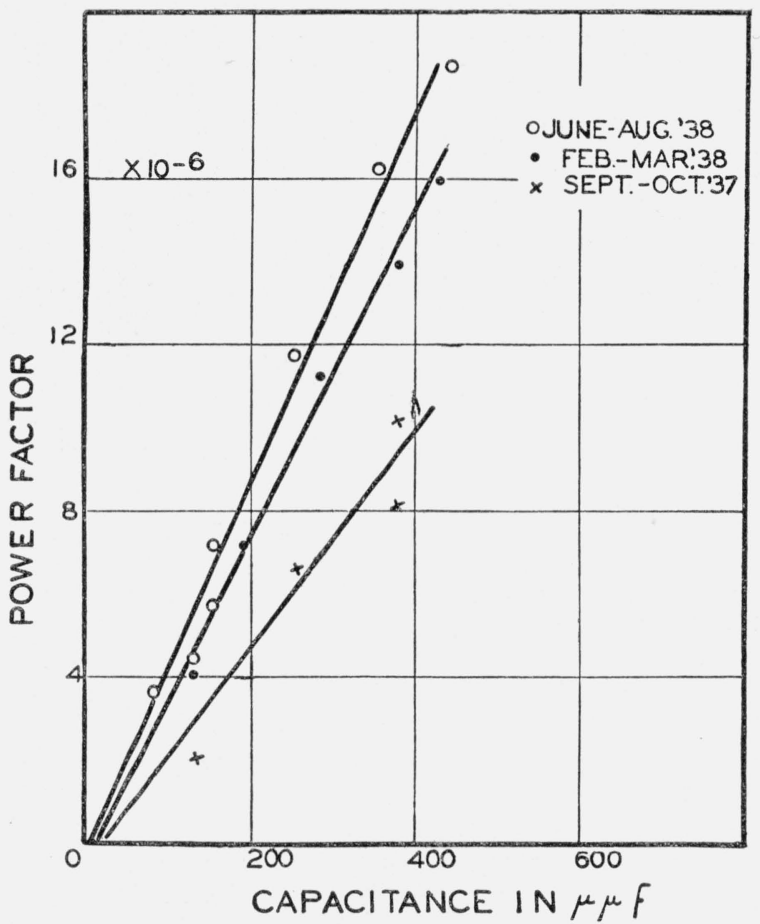

FIGURE 11.-Power-factor-capacitance curves of a guard-ring air capacitor with aluminum electrodes indicating increase in power factor with time due to aging of electrode surface.

The slopes of the power-factor-capacitance curves shown so far are functions of the areas of the electrodes. Since the power factors of a guard-ring capacitor are inversely proportional to the electrode separation, the product of power factor and electrode separation should give a term which is a function of the electrode material and the conditions of measurement and is independent of the geometry of the capacitor. Accordingly, this product has been used to express the power-loss measurements on the various electrode materials mentioned above. The results are listed in table 1 as a product of phasedefect angle (which is equivalent to power factor) in microradians and electrode separation in millimeters for eight different relative humidities and three frequencies of measurement. In every case, except for the anodized aluminum, the power factor reaches a maximum between 43 and 75 percent relative humidity, and in general the maximum occurs at a higher humidity at the higher frequencies. In addition, where data at 97 percent are available there appears to be a minimum at about 85 percent relative humidity. The data from the table for aluminum and silver-plated electrodes are shown graphically in figure 12 . 
TABLE 1.-Effect of electrode material and relative humidity upon the power factor (phase defect angle) of a guard-ring air capacitor

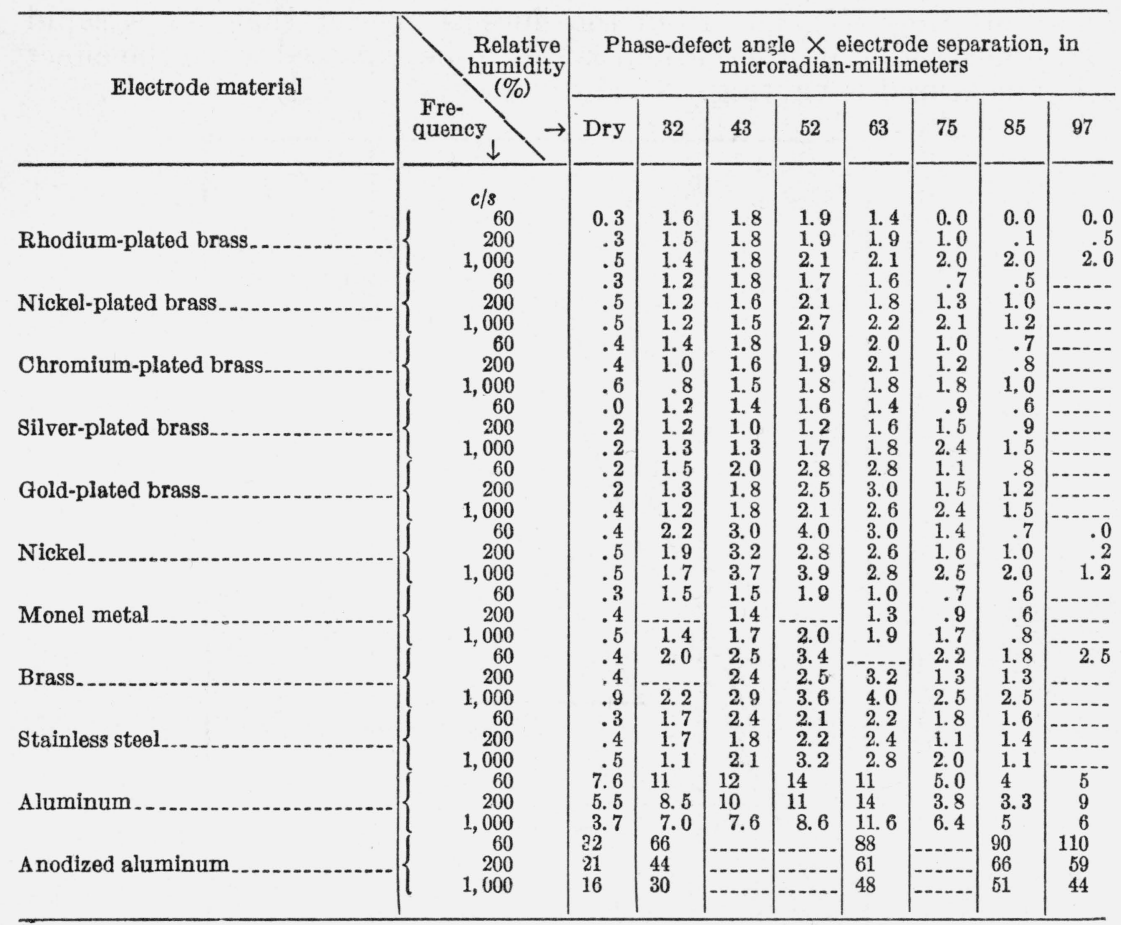

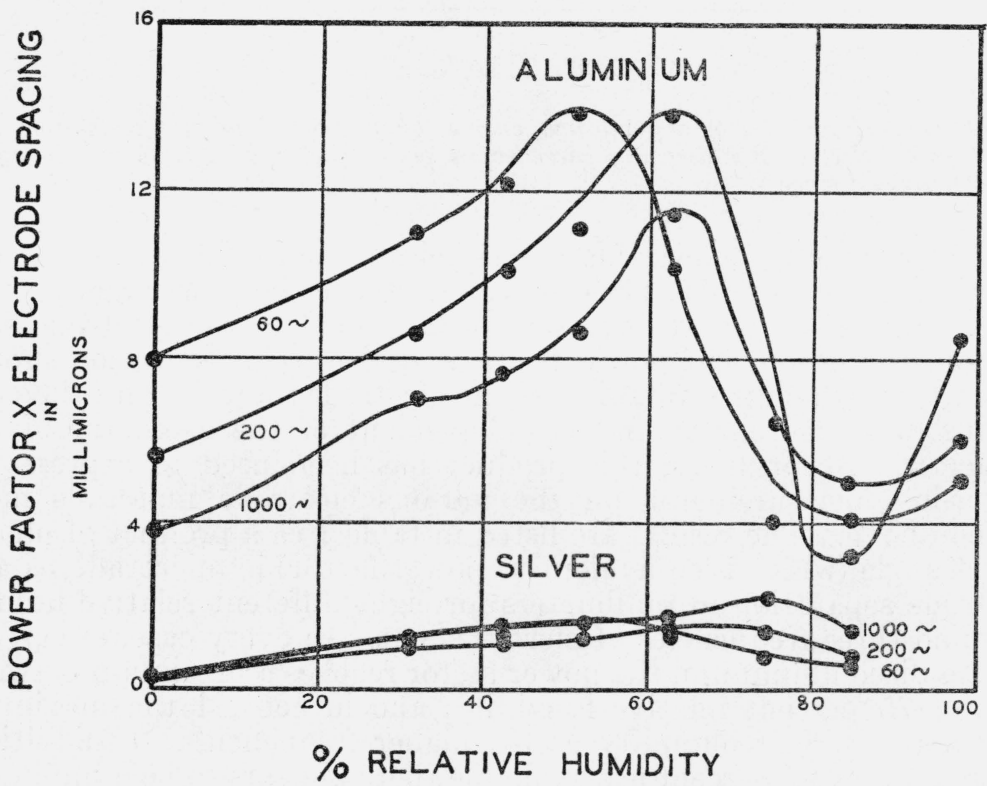

FIGURE 12.-Losses in guard-ring air capacitors with aluminum and silver electrodes as a function of relative humidity. 
A further examination of table 1 indicates that from the point of view of low power factor, aluminum is definitely the poorest of the materials listed for the electrodes of an air capacitor. This is to be expected because of the relatively thick oxide layer on metallic aluminum. Increasing the thickness of the layer by anodizing produces a marked increase in the observed power factors. Among the remaining materials there is little choice, the silver-plated electrodes showing the lowest power factors by a small margin. With these materials the observed losses appear to be almost entirely due to moisture because only a very slight effect was observed in the dry atmosphere.

In obtaining the above data it was found that cleaning the electrodes and keeping them clean was quite necessary for consistent results. Small quantities of dust were especially troublesome, particularly at high humidities and with electrode separations of less than a millimeter. Fortunately, by the use of polished or semipolished electrode surfaces it was quite easy to detect the presence of dust. Next to dust, oil and grease films were found most likely to give abnormally high power factors. It was found, for example, that cleaning the electrodes with commercial grade carbon tetrachloride or benzene sometimes left an invisible oil film which considerably increased the power factor of the capacitor. Cleansing with soap and water with adequate rinsing was found to give the cleanest surfaces from the point of view of low power factor.

\section{CONTRIBUTION OFA SURFACE LAYER TO POWER FACTOR}

Inasmuch as the losses in a guard-ring air capacitor appear to occur in surface layers on the electrodes, it is desirable to determine analy tically the effect of such layers on the power factors. In figure 13 are shown (a) a section of the electrodes of a capacitor separated by a distance, $d$, and with a film on each electrode of thickness, $t$, (b) a circuit diagram of the arrangement showing a perfect capacitor, $C_{1}$, in series with a partially conducting capacitor of capacitance and resistance, $C_{2}$ and $R_{2}$ (the thickness of the dielectric of $C_{2}$ will be twice that of the film), and (c) an equivalent circuit of capacitance, $C$, and resistance, $R$. The capacitance, $C$, and power factor, $\phi$, of the equivalent circuit are, when $t<<d$ or $C_{1}<<C_{2}$ and second-order terms are neglected,

$$
\begin{aligned}
& C=C_{1}\left(1-\frac{\omega^{2} R_{2}^{2} C_{1} C_{2}}{1+\omega^{2} R_{2}^{2} C_{2}^{2}}\right) \\
& \phi=\frac{\omega R_{2} C_{1}}{1+\omega^{2} R_{2}^{2} C_{2}^{2}},
\end{aligned}
$$

where $\omega$ is $2 \pi$ times the frequency, $f$. If the dielectric constant in esu and conductivity in $\mathrm{ohm}^{-1} \mathrm{~cm}^{-1}$, of the film are $\epsilon$ and $\sigma$, respectively, the above equations may be written

$$
C=C_{1}\left[1-\frac{t}{d} \times \frac{2 \epsilon\left(5.5 \times 10^{-13} \frac{f}{\sigma}\right)^{2}}{1+\left(5.5 \times 10^{-13} \frac{f \epsilon}{\sigma}\right)^{2}}\right] \equiv C_{1}\left[1-\frac{t}{d} \times N\right]
$$


and

$$
\phi=\frac{t}{d} \times \frac{1.11 \times 10^{-12} \frac{f}{\sigma}}{1+\left(5.5 \times 10^{-13} \frac{f \epsilon}{\sigma}\right)^{2}} \equiv \frac{t}{d} \times M
$$

The numerical factors arise from expressing the capacitance in practical units. In these equations the capacitance change and power factor are linear functions of the $t / d$ ratio and of factors $N$ and $M$,

(a)

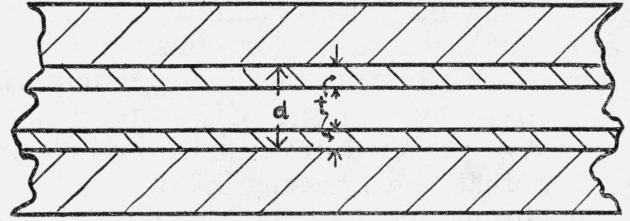

$\mathrm{C}_{2}$

(b)

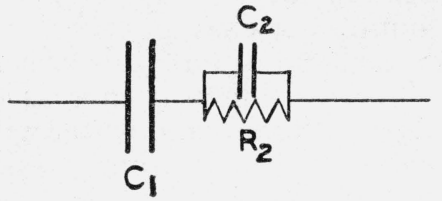

(c)

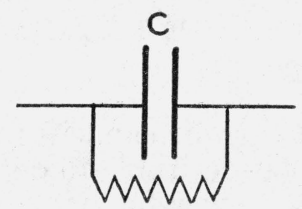

$\mathrm{R}$

FigURE 13.-Electrical representation of an electrode surface layer.

In (a) is shown a section of the electrodes of a capacitor separated by a distance, $d$, with films on each electrode surface of thickness, $t$. In $(b)$ is shown the circuit including the electrode film which can be reduced to the equivalent circuit (c). respectively, which are in turn functions of $f / \sigma$ and $\epsilon$. The inversely proportional relation of eq 4 between power factor and electrode separation is in accordance with the experimental data for guard-ring capacitors. The manner in which the factors $N$ and $M$ vary with $f / \sigma$ is shown in figure 14 with $\epsilon$ as a parameter. Here $N$ and $\log _{10} M$ are plotted against $\log _{10} f / \sigma$ for values of $\epsilon=1,10$, and 100 . The $M$ curves show that a film on an electrode surface produces a maximum power-factor effect for critical values of $f / \sigma$. The ratio $f / \sigma$ is chosen as a variable rather than $f$, because in general $\sigma$ will vary appreciably with $f$, thus making it impossible to consider $f$ as a variable with $\sigma$ constant. The value of this ratio which produces the greatest power factor is

$$
\frac{f}{\sigma}=\frac{1.8 \times 10^{12}}{\epsilon}
$$

and the corresponding power factor is

$$
\phi_{(\max )}=\frac{t}{d \epsilon} .
$$

Since $\epsilon$ will always be greater than unity, the maximum possible powerfactor effect of a thin film on an electrode surface will always be less than the ratio of the film thickness to the electrode separation. Attention should be called to the fact that the maximum is independent of either the conductivity of the film or the frequency. ${ }^{3}$

${ }^{3}$ Equations 5 and 6 are based on the assumption that $\epsilon$ is independent of $f / \sigma$. Although this may not be strictly true, it is not likely that the variation of $\mathrm{e}$ will be sufficiently great to appreciably affect the value of $f / \sigma$ at which the maximum of $\phi$ occurs. 
As the power factor passes through its maximum value with varying conductivity and frequency, the capacitance, $C$, changes from $C_{1}$ to

$$
C=C_{1}\left[1-\frac{2 t}{\epsilon d}\right],
$$

as shown by the $N$ curves of figure 14. These types of simultaneous variations of capacitance and power factor with frequency are wellknown dielectric phenomena occurring with polar molecules in a viscous medium [8] and in a heterogeneous dielectric [9] such as was

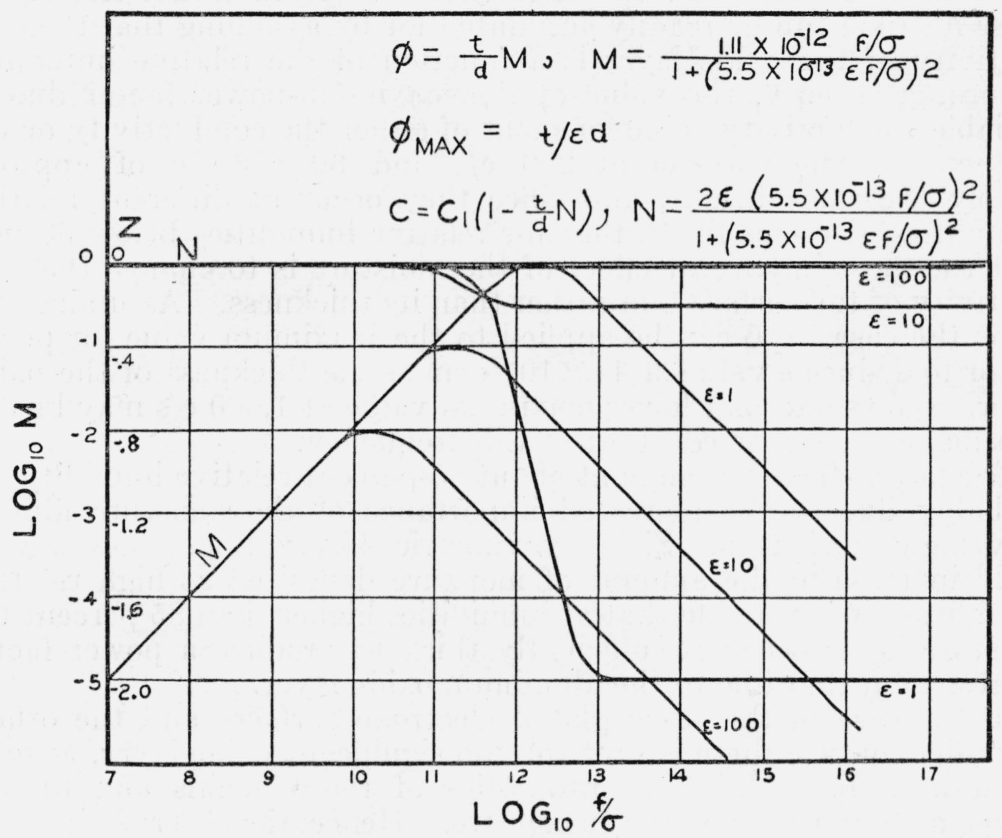

FIGURE 14.-Curves showing the effect of an electrode film of thickness, $t$, conductivity, $\sigma$, and dielectric constant, $\epsilon$, on the power factor, $\phi$, and capacitance, $C$, of a guard-ring air capacitor of electrode spacing, $d$; frequency is denoted by $f$.

proposed by Maxwell to explain certain types of dielectric absorption. Actually the electrode-film idea may be considered as a special case of the latter reference.

If the power-factor maxima and the over-all change of capacitance with frequency were determined for a guard-ring capacitor with an electrode surface layer, the thickness, dielectric constant, and conductivity of the layer could be determined by applications of eq 5,6 , and 7 . Unfortunately, the data obtained in this investigation are inadequate for these computations. The frequency range is not sufficient to obtain the power-factor maxima and the mechanical stability of the capacitors was not sufficient to determine the capacitance changes with the required accuracy. However, the power-factor maxima obtained with varying humidities are subject to explanation by the above equations, and an examination of the data with respect to 146065-39-5 
these equations yields some information concerning the nature of the electrode films.

According to gravimetric observations [10], the amount of moisture taken up by a metal surface increases continuously with relative humidity, and above 85 percent the increase is very rapid. If the moisture formed a layer on the electrode of regularly increasing thickness, and if the conductivity of the layer were independent of thickness the observed power factors would increase with relative humidity in the same manner as does the amount of moisture deposited. Such a continuous increase is not in accordance with the power-factor maxima shown in figure 12. For the case of aluminum electrodes, these maxima can be readily accounted for by assuming that the conductivity of the oxide layer is a function of the relative humidity. According to eq 6, the value of the maximum-power factor due to variable conductivity is independent of either the conductivity or the frequency. The maxima at $200 \mathrm{c} / \mathrm{s}$ and $60 \mathrm{c} / \mathrm{s}$ are of approximately the same height, and since they occur at different relative humidities, it seems likely that for relative humidities below 80 percent the most important effect of the moisture is to change the conductivity of the surface film rather than its thickness. Assuming this to be the case, eq 6 can be applied to the maximum value for power factor to deduce a value of $14 \times 10^{-7} \epsilon \mathrm{cm}$ as the thickness of the oxide layer. The somewhat lower maximum value at $1,000 \mathrm{c} / \mathrm{s}$ may be due to a higher dielectric constant at this frequency.

The power-factor minima at about 85-percent relative humidity are probably due to the increased importance of the moisture film at very high humidities. Since gravimetric observations show a very rapid increase in the amount of moisture deposited at high relative humidities, it is possible that at humidities higher than 85 percent the moisture layer becomes sufficiently thick to produce a power factor greater than that due to the aluminum oxide layer.

In the case of the silver-plated electrode surfaces and the others of similar nature there is probably no significant oxide layer, as indicated both by well-known properties of these metals and by the power-factor data for a dry atmosphere. Hence, the observed increase in power factor with relative humidity must be due entirely to the moisture on the electrode surfaces. An explanation of the maxima requires that the conductivity of the layer be a function of its thickness, probably increasing as the thickness is increased.

If the moisture layer is alone responsible for the observed power factors it is necessary to assume conductivities considerably below those obtained for water in conductivity cells. These latter values range upward from approximately $1 \times 10^{-6} \mathrm{ohm}^{-1} \mathrm{~cm}^{-1}$. Referring to figure 14 , it may be seen that such conductivities would give, at commercial and audio frequencies, extremely small power-factor effects unless the film were very thick. For example, with a conductivity of $1 \times 10^{-6} \mathrm{ohm}^{-1} \mathrm{~cm}^{-1}$ and at a frequency of $60 \mathrm{c} / \mathrm{s}$, a film thickness of 30 microns would be required to produce the maximum power factor shown in figure 12. This is about ten thousand times the thickness of water layers observed on such metals by gravimetric methods [10]. On the other hand, the conductivity necessary to produce the maximum power factor can be computed by eq 5 if the dielectric constant and frequency are known. Assuming a dielectric constant of 80 , the power-factor maximum at $60 \mathrm{c} / \mathrm{s}$ is produced by a conduc- 
tivity of $2.6 \times 10^{-9} \mathrm{ohm}^{-1} \mathrm{~cm}^{-1}$, which is considerably less than the normal conductivity of water. Equation 5 also shows that the conductivity of an electrode film necessary to produce a power-factor maximum increases with increasing frequency. Since the maxima at higher frequencies occur at higher humidities, the data and theory are consistent in assuming the conductivity of the moisture layer to increase with increasing thickness of the layer. Further evidence that the observed power factors for all electrode metals except aluminum are due primarily to the moisture layer is found in the fact that in general the magnitude of the maximum power factor is greater at the higher frequencies. This is essential because these maxima occur at higher humidities at the higher frequencies.

Assuming the dielectric constant of the moisture film to be 80 , and applying eq 6 to the maximum power factors for silver electrodes, as shown in figure 12 , values of $13 \times 10^{-6} \mathrm{~cm}$ and $19 \times 10^{-6} \mathrm{~cm}$ are obtained as the thicknesses of the film at relative humidities of 52 and 75 percent, respectively. These thicknesses are about 10 times the values obtained gravimetrically but this discrepancy may be due to the assumed value for the dielectric constant of the layer. A dielectric constant of 8 for the moisture layer would make the data consistent with gravimetric observations. More complete data would, as pointed out above, give both the dielectric constant and conductivity of the layer making it unnecessary to assume these values from other types of measurements. Additional data would also furnish more complete evidence as to whether the power factors observed with silver-surfaced and other similar electrodes are due to the moisture film alone or to moisture in combination with a layer of some foreign material. The present data are quite definite in indicating that the conductivity of the electrode film is appreciably less than the normal values for water, but can give only a ratio of film thickness to its dielectric constant. If upon separating these latter two quantities by further measurements it is found that the thicknesses are greater than those observed gravimetrically for water, then it would definitely appear that the electrode film had important constituents other than water. The conductivities necessary to produce a maximum power factor could then be attributed to a mixture of water and the other material of the film.

The data for anodized aluminum (table 1) show no definite powerfactor maxima with relative humidity. This would seem to indicate that the moisture does not penetrate the oxide layer sufficiently to cause a large enough average conductivity to produce a maximum.

\section{EFFECT OF A NONHOMOGENEOUS FIELD ON POWER FACTOR}

In order to determine the effect, if any, of a nonhomogeneous electric field on the power factor of an air capacitor, sharp-edged, annular grooves were cut in one of the electrodes of a brass guard-ring capacitor, as shown in figure 15 . With such a capacitor there is an appreciably nonuniform field between the electrodes. The power factor of this capacitor was then measured at various electrode spacings and relative humidities. The results at $60 \mathrm{c} / \mathrm{s}$ are shown in figure 16 with power factor plotted against capacitance with relative humidity as a parameter. The dashed line, which represents the results at 43-percent 
relative humidity for smooth brass electrodes (shown previously in fig. 9), is shown for comparison. The increase in power factor due to the nonhomogeneous field is quite pronounced. It is also evident that the power factor increases appreciably with relative humidity in

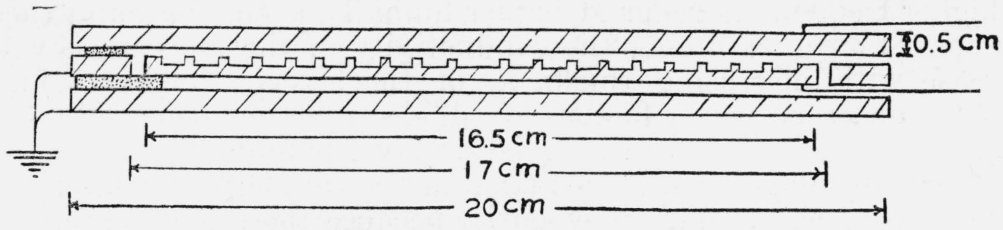

FIGURE 15.-Section of a guard-ring capacitor showing grooves cut in one of the brass electrodes to produce a nonhomogeneous field.

the low-humidity range and then drops to low values at high humidities. This latter behavior is qualitatively similar to that observed when the field of the guard-ring capacitor was uniform. On the other hand, there is to be noted in figure 16 a departure of the power-factorcapacitance curve from proportionality at 18-percent relative humidity and from linearity at 85 -percent relative humidity. These curves

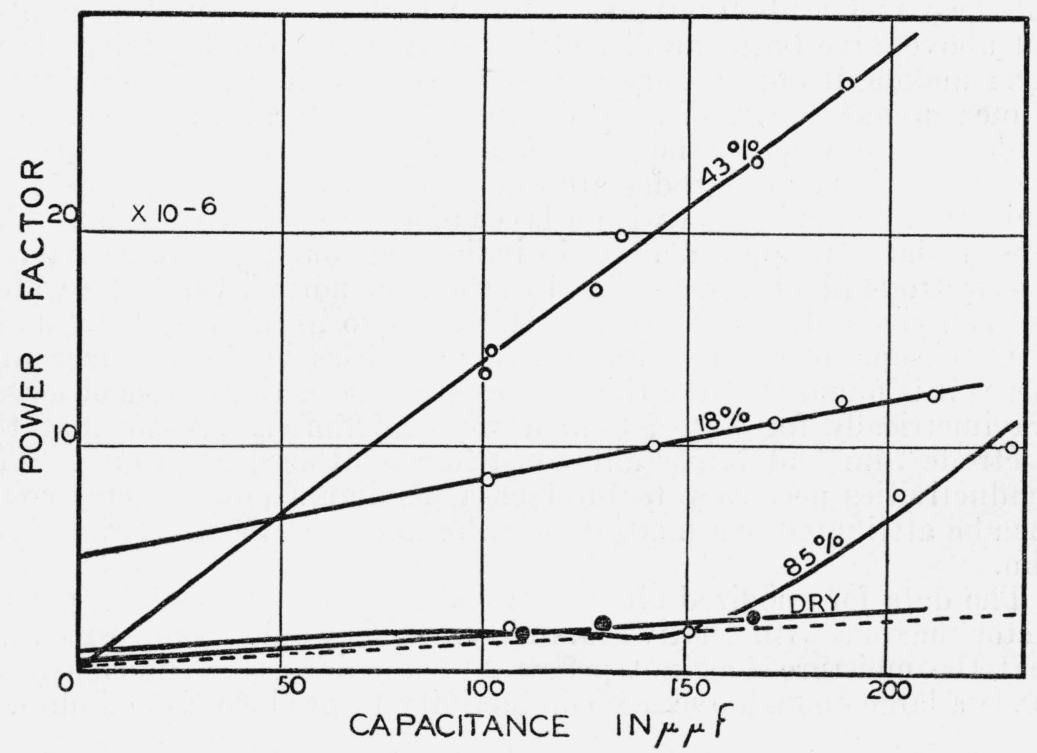

FigURE 16.-Power factors of a guard-ring capacitor with nonhomogeneous field at various humidities.

The dashed line represents the equivalent values for a guard-ring capacitor with a uniform field at 43-percent relative humidity. Measurements were at $60 \mathrm{c} / \mathrm{s}$.

may be due to one or both of the following factors: (1) the capacitance of the grooved-electrode capacitor is not inversely proportional to its electrode separation, and (2) the degree of nonuniformity of the field varies with the capacitance or electrode separation.

It is probable that the power factors represented in figure 16 are also due to energy losses in a layer on the electrode surface because of the fact that power-factor maxima with variable relative humidity 
are observed. If corona were a cause of the increase in power factor produced by field distortion, there would very likely be no maximum with relative humidity. Further evidence that measurable corona losses were not present was found in the independence of the power factor upon the voltage applied to the capacitor. Although it was difficult to estimate the maximum potential gradient in the capacitor, the average field was about $500 \mathrm{v} / \mathrm{cm}$. Upon increasing this value to $1,200 \mathrm{v} / \mathrm{cm}$, no change in power factor was observed. The increase in power factor caused by the nonuniform field may be attributable to the fact that in such a field, dipoles as well as ions would undergo a translatory motion.

The question which now aptly arises is, how much of the loss observed in the ordinary guard-ring capacitors was due to the slight nonhomogeneity of field at the gap between the measuring electrode and the guard ring. Tests have been made on guard-ring capacitors with brass electrodes in which the width of the guard-ring gap varied from 0.2 to $2 \mathrm{~mm}$, with a resulting variation in the degree of nonhomogeneity. No appreciable differences in power factors were observed, indicating that the effect of nonhomogeneity in the guardring capacitors used for obtaining the data of table 1 was quite negligible.

It is probable that the observed power factor of the three-electrode rotating-plate capacitor, previously discussed, is due to the nonhomogeneous field at the edges of its electrodes. Referring back to figure 1 , it is seen that the power factor of this capacitor, $C_{s}$, decreases with increasing capacitance. The ratio of nonuniform field to uniform field in a rotating-electrode capacitor decreases as the capacitance increases, hence the part of the power factor originating in the nonuniform field at the edges of the electrodes of such a capacitor would decrease with increasing capacitance. Although qualitatively similar relations between power factor and capacitance were obtained for two-electrode capacitors when there was conductance between the measuring electrodes, such cannot be the cause of the power factor of the three-electrode capacitor, because the guard electrode makes possible the complete elimination of such an effect. Nor is it likely that the power factors represented by the $C_{s}$ curves of figure 1 are due to an electrode surface layer of variable thickness or conductivity, because (1) the power-factor variation with capacitance is too uniform to be due to an irregular surface layer, and (2) the same type of variation has been obtained with both aluminum electrodes and nickelplated brass electrodes in the capacitor. The three-electrode rotating-plate capacitor was originally built with aluminum electrodes and its power-factor characteristic was believed to be due to an irregular surface layer. When it was found that aluminum electrodes gave the largest power factor of the common electrode metals the capacitor was rebuilt with nickel-plated brass electrodes. Although the power factor of the rebuilt capacitor was reduced, the same type of variation with capacitance was obtained, indicating that some source other than surface irregularities must be the cause of its power-factor characteristic. The $C_{s}$ curves of figure 1 represent nickel-plated electrodes, approximately $3 \mathrm{~mm}$ apart when interleaved. Referring to table 1 , it is noted that the power factor due to such electrodes at $3-\mathrm{mm}$ spacing should always be less than $10^{-6}$ when in a uniform field. It is therefore concluded that the power-factor values shown in figure 1 
for the three-electrode rotating-plate capacitor are on account of the nonhomogeneous field at the edges of the electrodes.

As should be expected, it was found that the power factor of the rotating-plate capacitor varied considerably with humidity. It was possible to maintain reasonably constant power-factor values from day to day by directing a gentle stream of dry air through the capacitor. The curves of figure 1 were taken under such conditions.

\section{CONCLUSION}

1. The power factors of most variable two-electrode air capacitors may be represented in the form $A+B / C$, where $C$ is the capacitance, $A$ depends upon the electrode material, frequency, and humidity, and $B$ depends upon the insulation material, frequency, and humidity.

2. The losses in two-electrode capacitors are appreciably dependent on the relative humidity and frequency. Specifications of power factor for such capacitors are quite meaningless unless the capacitor is in equilibrium with its surrounding atmosphere and the conditions of measurement are clearly defined.

3. The power factor of a guard-ring capacitor is proportional to its capacitance (inverse electrode separation). The losses are also an important function of relative humidity, showing maximum values in the low-frequency range for relative humidities of about 50 percent.

4. Guard-ring capacitors with aluminum electrodes have appreciably greater power factors than those of other materials. Other materials tested were stainless steel, monel metal, nickel, and brass in addition to brass plated with silver, nickel, chromium, gold, and rhodium. No noteworthy differences between the power factors of capacitors of these materials were observed, although the silverplated electrodes appeared to be superior by a slight margin.

5. The losses observed in guard-ring capacitors may be explained by a layer of oxide or water or both on the electrode surface. The contribution of such a layer to the power factor of the capacitor will be a maximum when the conductivity, $\sigma$, and dielectric constant, $\epsilon$, of the layer are related to the frequency, $f$, as

$$
\frac{f}{\sigma}=\frac{1.8 \times 10^{12}}{\epsilon}
$$

The maximum possible power factor from such a layer for variable $f$ and/or $\sigma$ is

$$
\phi_{(\max )}=\frac{t}{d \epsilon},
$$

where $t$ is thickness of the layer and $d$ the electrode separation.

6 . The data indicate that (1) the conductivity of moisture films on metals increases as the film thickness is increased and, (2) both the conductivity and dielectric constant of the moisture films are appreciably less than those of water as ordinarily measured.

7. The contribution of a surface layer to power factor is appreciably increased when the electric field is nonuniform. This may give rise to a power factor in a three-electrode rotating-plate capacitor which decreases with increasing capacitance. 


\section{REFERENCES}

[1] R. F. Field and D. B. Sinclair, Proc. Inst. Radio Engrs. 24255 (1936). This paper contains a good bibliography on previous work on losses in air capacitors.

[2] A. V. Astin, J. Research NBS 21, 425 (1938) RP1138.

[3] J. C. Balsbaugh and Alfred Herzenberg, J. Franklin Inst. 218, 49 (1934).

[4] W. B. Kouwenhoven and E. L. Lotz, Am. Inst. Elec. Engrs. Trans. 57, 766 (1938).

[5] General Radio Experimenter (October 1935).

[6] J. G. Chaffe, Proc. Inst. Radio Engrs. 22, 1009 (1934).

[7] Cir. BS C74 (1918).

[8] P. Debye, Polar Molecules (The Chemical Catalog Co., New York, N. Y., 1929).

[9] L. Hartshorn, J. Int. Elec. Eng. 64, 1152 (1926).

[10] Stromberg, Kgl. Svenska Vetenskapsakad Handl. 6, 113.

Washington, March 2, 1939. 